\title{
New prescriptions of turbulent transport from local numerical simulations
}

\author{
V. Prat ${ }^{1,2,3}$, F. Lignières ${ }^{2,3}$ and G. Lesur ${ }^{4,5}$ \\ ${ }^{1}$ MPA, Karl-Schwarzschild-Str. 1, 85748, Garching b. München, Germany \\ email: vprat@mpa-garching.mpg.de \\ ${ }^{2}$ Université de Toulouse; UPS-OMP; IRAP; Toulouse, France \\ ${ }^{3}$ CNRS; IRAP; 14 , avenue Édouard Belin, F-31400 Toulouse, France \\ ${ }^{4}$ Univ. Grenoble Alpes, IPAG, 38000, Grenoble, France \\ ${ }^{5}$ CNRS, IPAG, 38000, Grenoble, France
}

\begin{abstract}
Massive stars often experience fast rotation, which is known to induce turbulent mixing with a strong impact on the evolution of these stars. Local direct numerical simulations of turbulent transport in stellar radiative zones are a promising way to constrain phenomenological transport models currently used in many stellar evolution codes. We present here the results of such simulations of stably-stratified sheared turbulence taking notably into account the effects of thermal diffusion and chemical stratification. We also discuss the impact of theses results on stellar evolution theory.
\end{abstract}

Keywords. Hydrodynamics, turbulence, stars: evolution, stars: rotation

\section{Introduction}

Transport of chemical elements and transport of angular momentum both have a strong impact on stellar structure and evolution and can be done either by microscopic processes, which are relatively well known, or by macroscopic motions, which are still poorly understood. In particular, as massive stars often experience fast rotation, rotationally induced motions may play a crucial role. These motions are usually decomposed into large-scale axisymmetric motions, such as differential rotation and meridional circulation, and small-scale turbulent motions generated by various instabilities. Whereas the former can be either directly solved in 2D stellar evolution codes or modelled in 1D codes thanks to additional assumptions (e.g. the shellular model of Zahn 1992), the latter are fundamentally three-dimensional and thus need to be modelled in both 1D and 2D stellar evolution codes. The main obstacle to building such models is that these turbulent motions have very small length and time scales as compared to evolutionary ones.

In many current stellar evolution codes, transport of chemical elements due to shear instability is taken into account thanks to a set of diffusion coefficients initially derived by Zahn (1992) from phenomenological arguments. One of these coefficient, the radial transport coefficient generated by radial differential rotation, is given in Zahn's model by

$$
D_{\mathrm{t}}=\frac{\kappa}{3} \frac{\mathrm{Ri}_{\mathrm{c}}}{N^{2}}\left(r \sin \theta \frac{\mathrm{d} \Omega}{\mathrm{d} r}\right)=\frac{\kappa}{3} \frac{\mathrm{Ri}_{\mathrm{c}}}{\mathrm{Ri}},
$$

where $\kappa$ is the thermal diffusivity of the fluid, very large in stellar interiors; $\mathrm{Ri}=$ $(N / S)^{2}$ the Richardson number, which compares stratification, characterised by the Brunt-Väisälä frequency $N$, and shear, characterised by the shear rate $S=r \sin \theta \mathrm{d} \Omega / \mathrm{d} r$; $\mathrm{Ri}_{\mathrm{c}}$ its critical value; $r, \theta$ the spherical coordinates and $\Omega$ the angular velocity of the star. 
The usual way to constrain models of turbulent transport is to compare measurements of surface chemical abundances to predictions of stellar evolution models. Recently, it has become possible to constrain the internal rotation profile of red (sub-)giant stars using asteroseismology (e.g. Deheuvels et al. 2012). Here we use another approach: we perform local direct numerical simulations of steady homogeneous stably stratified sheared turbulence to test existing models and propose new prescriptions for stellar evolution codes.

Our governing equations are presented in Sect. 2. In Sect. 3 we summarize the results we have obtained concerning the turbulent chemical diffusity and in Sect. 4 we present new results about turbulent thermal diffusivity and turbulent viscosity. Finally, we conclude on our results and prospects in Sect. 5 .

\section{Governing equations}

Our mean flow configuration consists of a uniform vertical velocity shear and stable, uniform vertical temperature and concentration gradients. We solve the dimensionless form of the Boussinesq equations

$$
\begin{aligned}
\vec{\nabla} \cdot \vec{v} & =0, \\
\frac{\partial \vec{v}}{\partial t}+(\vec{v} \cdot \vec{\nabla}) \vec{v} & =-\vec{\nabla} p+\left(\operatorname{Ri} \theta+\operatorname{Ri}_{\mu} c^{\prime}\right) \vec{e}_{z}+\frac{1}{\operatorname{Re}} \Delta \vec{v}, \\
\frac{\partial \theta}{\partial t}+\vec{v} \cdot \vec{\nabla} \theta+v_{z} & =\frac{1}{\mathrm{Pe}} \Delta \theta, \\
\frac{\partial c^{\prime}}{\partial t}+\vec{v} \cdot \vec{\nabla} c^{\prime}+v_{z} & =\frac{1}{\mathrm{Pe}_{\mathrm{c}}} \Delta c^{\prime},
\end{aligned}
$$

where $\vec{v}$ and $p$ denotes velocity and pressure, $\theta$ and $c^{\prime}$ temperature and concentration fluctuations around the mean profiles, $\mathrm{Ri}_{\mu}$ the chemical equivalent of the Richardson number, $\mathrm{Re}=U L / \nu$ the Reynolds number characterising the viscosity $\nu$ and $\mathrm{Pe}_{\mathrm{c}}=$ $U L / D_{\mathrm{m}}$ the chemical Péclet number characterising the molecular diffusivity $D_{\mathrm{m}}$. The regime of very high thermal diffusivities is explored using the so-called small-Pécletnumber approximation (SPNA, see Lignières 1999), in which Eqs. (2.2) and (2.3) are replaced by

$$
\begin{aligned}
\frac{\partial \vec{v}}{\partial t}+(\vec{v} \cdot \vec{\nabla}) \vec{v} & =-\vec{\nabla} p+\left(\operatorname{Ri} \operatorname{Pe} \psi+\operatorname{Ri}_{\mu} c^{\prime}\right) \vec{e}_{z}+\frac{1}{\operatorname{Re}} \Delta \vec{v}, \\
v_{z} & =\Delta \psi,
\end{aligned}
$$

noting $\psi=\theta / \mathrm{Pe}$.

\section{Turbulent chemical diffusivity}

Here we are interested in the turbulent chemical diffusivity $D_{\mathrm{t}}$. In the chemically neutral case $\left(\mathrm{Ri}_{\mu}=0\right)$, we studied its dependence on thermal diffusivity (see Sect. 3.1). Then, we investigated the effect of chemical stratification in the SPNA, as presented in Sect. 3.2.

\subsection{Effect of thermal diffusion}

We performed a series of simulations with different Péclet number in the Boussinesq approximation and one simulation in the SPNA. In the small-Péclet-number regime, we showed (Prat \& Lignières 2013) that the quantity $D_{\mathrm{t}} /\left(\kappa \mathrm{Ri}^{-1}\right)$ tends to a constant value 


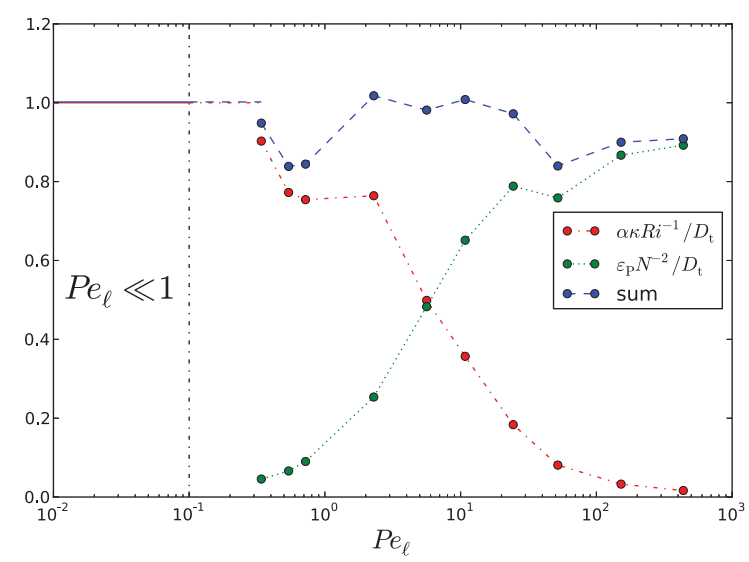

Figure 1. Ratios between models and effective diffusion coefficient as a function of the turbulent Péclet number $\mathrm{Pe}_{\ell}=u \ell / \kappa$, where $u$ and $\ell$ are turbulent velocity and length scales (Prat \& Lignières 2014)

as the Péclet number decreases, that is sensibly the same as in the SPNA. We can thus write

$$
D_{\mathrm{t}}=\alpha \kappa \mathrm{Ri}^{-1}
$$

with a constant $\alpha$. This is in agreement with Zahn's model (1.1). Our estimate of the proportionality constant, around $5.6 \times 10^{-2}$, is of the same order of magnitude as what Zahn (1992) proposed, but $30 \%$ smaller.

In the large-Péclet-number regime (see Prat \& Lignières 2014), the generalisation of Zahn's model proposed by Maeder (1995), intended to be also valid in this regime, turned to be incompatible with our simulations. In the opposite, we found a good agreement with the model of Lindborg \& Brethouwer (2008), proposed in the geophysical literature, in which the diffusion coefficient is given by

$$
D_{\mathrm{t}}=\frac{\varepsilon_{\mathrm{P}}}{N^{2}},
$$

where $\varepsilon_{\mathrm{P}}$ is the dissipation rate of turbulent potential energy. As illustrated in Fig. 1, the total diffusion coefficient estimated in our simulations can be seen as the sum of Zahn's model and Lindborg's one. Moreover, according to Osborn \& Cox (1972), the diffusion coefficient (3.2) is equal to the turbulent thermal diffusivity $\kappa_{\mathrm{t}}$. One can thus write

$$
D_{\mathrm{t}}=\kappa_{\mathrm{t}}+\alpha \frac{\kappa}{\mathrm{Ri}}
$$

where a prescription for $\kappa_{\mathrm{t}}$ is still to be given (see Sect. 4).

\subsection{Effect of chemical stratification}

Our SPNA simulations performed with different values of the chemical Richardson number $\mathrm{Ri}_{\mu}$ (also presented in Prat \& Lignières 2014) show that the quantity $D_{\mathrm{t}} /\left(\kappa \mathrm{Ri}^{-1}\right)$ is well represented by an affine function of $\mathrm{Ri}_{\mu}$, as represented in Fig. 2. As a consequence, one can write

$$
\frac{D_{\mathrm{t}}}{\kappa \mathrm{Ri}^{-1}}=\beta\left(\mathrm{Ri}_{\mathrm{c}}-\mathrm{Ri}_{\mu}\right),
$$

with $\beta=0.45$ and $\mathrm{Ri}_{\mathrm{c}}=0.12$. The turbulent diffusion coefficient is then given by

$$
D_{\mathrm{t}}=\beta \kappa \frac{\mathrm{Ri}_{\mathrm{c}}-\mathrm{Ri}_{\mu}}{\mathrm{Ri}},
$$




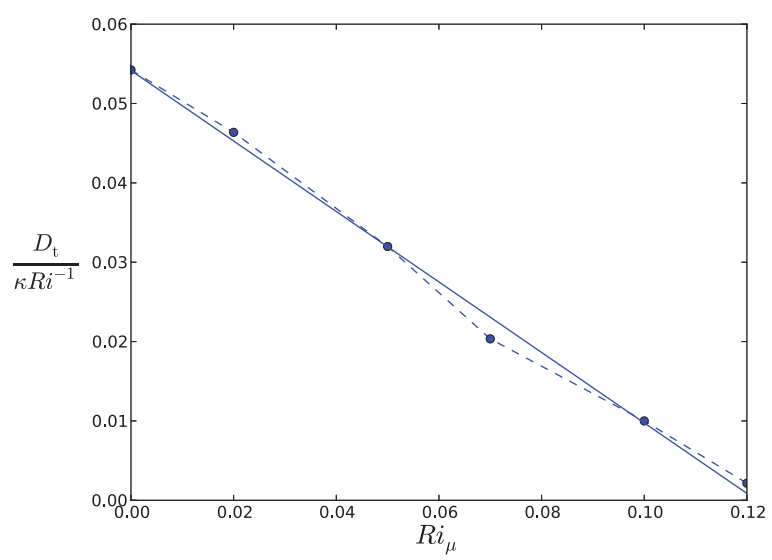

Figure 2. $D_{\mathrm{t}} /\left(\kappa \mathrm{Ri}^{-1}\right)$ as a function of the chemical Richardson number. Dots correspond to simulations and the solid line to the linear regression (Prat \& Lignières 2014).

which is of the same form as what Maeder \& Meynet (1996) derived in the small-Pécletnumber regime assuming that the stability criterion in presence of a $\mu$-gradient is

$$
\mathrm{RiPe}_{\ell}+\mathrm{Ri}_{\mu}>\mathrm{Ri}_{\mathrm{c}} .
$$

According to this criterion, chemical stratification is able to completely inhibit transport in regions of strong stable chemical gradients, such as around convective cores. In contrast, the model of Maeder (1997), which is incompatible with our simulations, allows mixing in such regions and has a better fit with observations (Meynet et al. 2013), thus suggesting the existence of an additional transport process or physical ingredient.

\section{Turbulent thermal diffusivity and turbulent viscosity}

This section is dedicated to new results concerning other diffusion coefficient, namely the turbulent thermal diffusivity $\kappa_{\mathrm{t}}$ and the turbulent viscosity $\nu_{\mathrm{t}}$. As regards $\kappa_{\mathrm{t}}$, recent simulation results displayed in Fig. 3 suggest that at constant Reynolds number, turbulent thermal diffusivity scales as $\mathrm{Ri}^{-4 / 3}$. This preliminary result has to be verified, and in particular, the dependence on the Reynolds number is currently investigated.

Concerning $\nu_{\mathrm{t}}$, it is important to note that in many evolution codes, it is simply taken to be equal to $D_{\mathrm{t}}$. In our simulations, we observe between them the following relation:

$$
\nu_{\mathrm{t}} \simeq \frac{3}{4} D_{\mathrm{t}}
$$

which is valid for all $\mathrm{Re}, \mathrm{Pe}, \mathrm{Ri}$, and $\mathrm{Ri}_{\mu}$.

\section{Conclusion}

Our simulations enabled us to test existing models of turbulent transport in stellar radiative zones. In particular, we recovered Zahn's model and the model of Maeder \& Meynet (1996) (in presence of $\mu$-gradients) in the small-Péclet-number regime and the model of Lindborg \& Brethouwer (2008) in the large-Péclet-number regime. In addition, we are now about to be able to give new prescriptions for turbulent transport of heat and angular momentum. Again, the fact that recovered models have not the best fit with observations suggests that another significant source of mixing may exist, or that an essential physical ingredient is missing. 


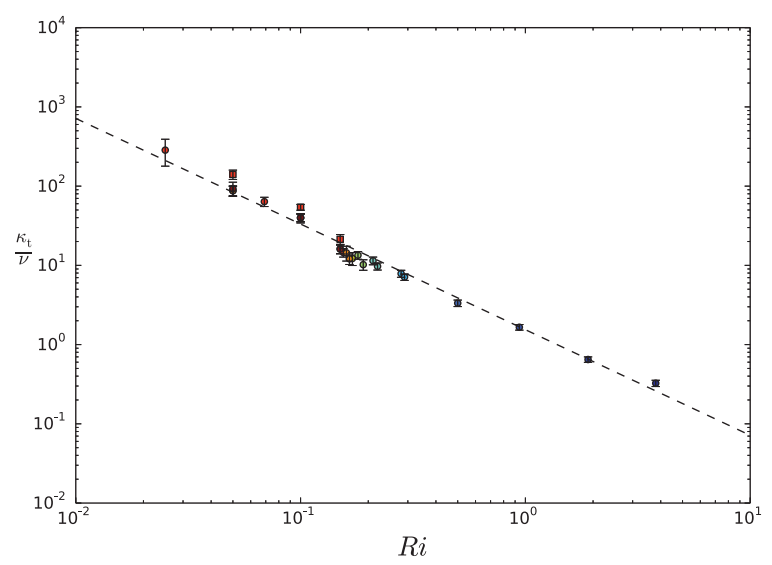

Figure 3. $\kappa_{\mathrm{t}} / \nu$ as a function of the Richardson number. Dots correspond to simulations at $\mathrm{Re}=10^{4}$ and Pe from $2 \cdot 10^{4}$ (brown) down to 15 (blue) and the dashed line to the power law $\propto \mathrm{Ri}^{-4 / 3}$.

To go further, one may think of studying the effect of a very efficient horizontal diffusion induced by horizontal differential rotation. As predicited by Talon \& Zahn (1997), this would weaken the effect of chemical stratification and thus enhance the transport. Other physical ingredients should be considered, such as the Coriolis force and the magnetic field. By altering shear instability or triggering new instabilities, they might increase the amount of mixing in stellar interiors.

Three-dimensional direct numerical simulations (DNS) can also be used to study other mixing processes, such as thermohaline convection or convective boundary mixing. Moreover, prescriptions from DNS could be used as sub-grid models to improve large-eddysimulations, that may be useful for non-local processes. Finally, all models coming from simulations must be included in stellar evolution codes to be compared to observations.

\section{References}

Deheuvels, S., García, R. A., Chaplin, W. J., et al. 2012, ApJ 756, 19

Lignières, F. 1999, A\&A 348, 933

Lindborg, E. \& Brethouwer, G. 2008, J. Fluid Mech. 614, 303

Maeder, A. 1995, A\&A 299, 84

Maeder, A. 1997, A\& $A$ 321, 134

Maeder, A. \& Meynet, G. 1996, A\&SA 313, 140

Meynet, G., Ekstrom, S., Maeder, A., et al. 2013, in Lecture Notes in Physics, Vol. 865, p. 3

Michaud, G. \& Zahn, J.-P. 1998, Theor. Comput. Fluid Dyn. 11, 183

Osborn, T. R. \& Cox, C. S. 1972, Geophys. Astrophys. Fluid Dynam. 3, 321

Prat, V. \& Lignières, F. 2013, A\&A 551, L3

Prat, V. \& Lignières, F. 2014, A\&SA 566, A110

Talon, S. \& Zahn, J.-P. 1997, A\&A 317, 749

Zahn, J.-P. 1992, A\&A 265, 115

\section{Discussion}

ARNETT: You said that your DNS calculations were "not turbulent". How would you defend their use for stellar dimensions?

PRAT: I said that when the effect of stratification is strong enough, we are not able to reach a turbulent statistical steady state because turbulence is decaying 
exponentially. The results I showed are based on turbulent simulations. About the validity of these results to stellar dimensions, I would like to emphasise the fact that the turbulent Reynolds number (i.e. based on turbulent length and velocity scales) is of the order of 100, as expected in stably stratified zones (see e.g. Michaud \& Zahn 1998).

Khalak: Could you comment why chemical diffusion will diminish chemical stratifications caused by thermal diffusion?

PRAT: What I told has not been correctly understood. Chemical stratification is not caused by thermal diffusion, and it is turbulent horizontal chemical diffusion that can weaken the effect of chemical stratification by smoothing vertical chemical fluctuations and hence decreasing the amplitude of the buoyancy force.

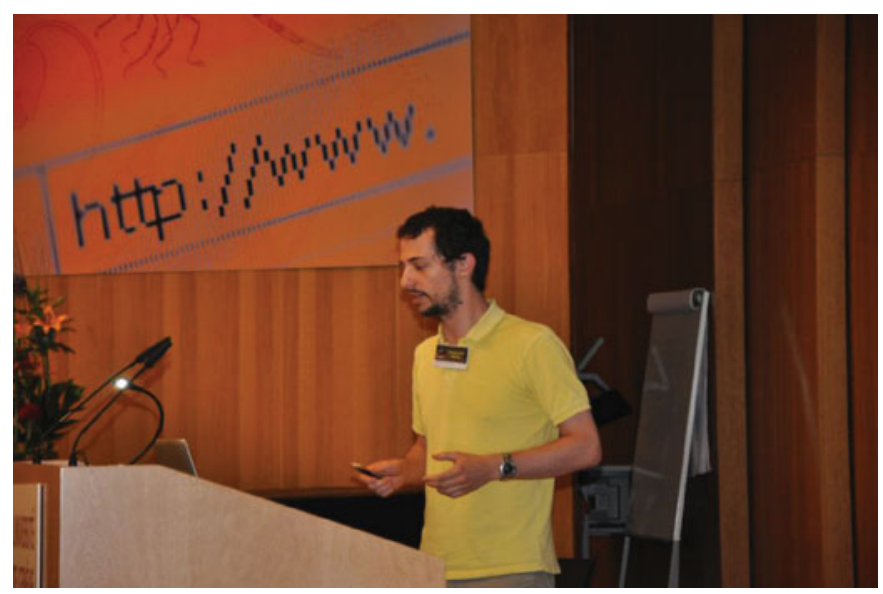

Vincent Prat

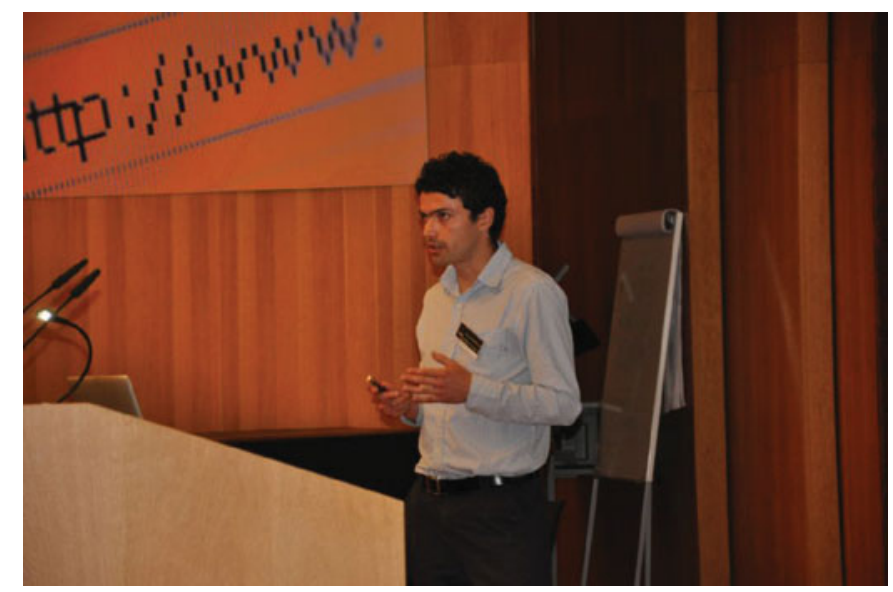

Oscar Hernan Ramìrez Agudelo 